\title{
Design and Construction of an Active Alignment Demonstrator for a Free-Space Optical Interconnect
}

\author{
G. C. Boisset, Member, IEEE, B. Robertson, and H. S. Hinton, Senior Member, IEEE
}

\begin{abstract}
An $x-y$ active alignment system based on Risley Beam Steerers is described. The demonstrator features a quadrant detector which detects the misalignment error between the center of a spot of light and the center of the quadrant detector. This misalignment error is then used by a new algorithm to calculate the rotational displacement required for the two Risley Beam Steerers to steer the spot of light to the center of the quadrant detector. The experimental results indicate that any spot misaligned by up to $160 \mu \mathrm{m}$ on the quadrant detector will be systematically centered by the demonstrator system.
\end{abstract}

\section{INTRODUCTION}

$\mathbf{F}$ REE-SPACE optical interconnects have the potential of alleviating the communication bottlenecks that will arise in future connection-intensive electronic systems [1]; however, to be of practical use, such interconnects must remain aligned for long periods of time in harsh industrial conditions. One way to maintain alignment is to design an extremely rigid system that will not drift over time. This can be performed by removing as many degrees of freedom as possible from the interconnect, [2] and by prealigning components. Another way to maintain registration is through the use of active alignment, a process in which system parameters such as throughput or error in spot position are monitored and fed back to a controller which realigns the system by altering the state of the optics. Such feedback loops exist in CD players, for example [3].

One area in which active alignment has tremendous potential is that of an optical backplane with removable Printed Circuit Boards (PCB's). This letter describes an $x-y$ active alignment demonstrator for a free-space optical link based on Risley Beam Steerers (RBS's) [2]. The closed loop system, outlined in Fig. 1, consists of: 1) a quadrant detector (QD) on which the alignment beam is incident and forms a spot 2) optical components 3 ) two stepper motors which rotate the RBS's in their optomechanical holders, and 4) a computer that samples the four signals originating from the $\mathrm{QD}$, computes the distance between the center of the spot and the center of the QD (the misalignment error), and computes the angular rotation necessary for two RBS's to center the spot.

Manuscript received December 9, 1994. This work was supported by the McGill NT/BNR-NSERC Industrial Chair in Photonic Systems.

G. C. Boisset and B. Robertson are with McGill University, Department of Electrical Engineering, 3480 University Street, Montréal, PQ, H3A 2A7, Canada.

H. S. Hinton was with McGill University, Department of Electrical Engineering, 3480 University Street, Montréal, PQ, H3A 2A7 Canada. He is now with the Department of Electrical and Computer Engineering, University of Colorado, Boulder, CO 80309-0425 USA.

IEEE Log Number 9410672.

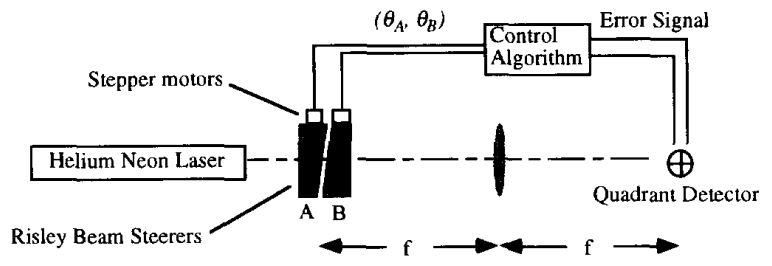

Fig. 1. Basic control loop of active alignment demonstrator.

\section{MODEL OF RBS MOVEMENT}

Glass wedges may be used to steer beams by imparting an angular displacement to them. These components, generally referred to as Risley Beam Steerers, have previously been used to provide $x-y$ alignment in free space-optical systems [2] and are a simple and cheap way of aligning free-space interconnects. Furthermore, components requiring rotational, as opposed to lateral, displacement can easily fit into a slotted plate, barrel or other integrated optomechanical setup. In addition, angular motion is easier to control than rectilinear motion when compensating for vibrational effects [4]. Two RBS's, RBS A and B with wedge angles $\beta_{A}$ and $\beta_{B}$ respectively, were chosen as the optical components to move in this demonstrator.

The effect of an RBS on a beam trajectory can be modeled as follows. From first order paraxial theory, the angular deviation, $\delta$, imparted by an RBS on a beam is $\delta=(n-1) \beta$, where $n$ is the index of refraction of the RBS. At the focal plane of a lens with focal length $f$, the lateral displacement, $r$, to the spot imparted by the RBS is:

$$
\begin{aligned}
& r_{A}=f \tan \delta_{A}=f \tan \left[(n-1) \beta_{A}\right] \\
& r_{B}=f \tan \delta_{B}=f \tan \left[(n-1) \beta_{B}\right] .
\end{aligned}
$$

Rotating the RBS about the $z$-axis will cause the spot to move along the circumference of a circle of radius $r$ at the focal plane of the lens. If RBS A and B are laid out in series along the beam path, the first order angular displacements imparted by the two RBS's will add. When RBS A and B are rotated about the $z$-axis, the spot will move on the periphery of a first circle of radius $r_{A}$ whose center is located on the periphery of a second circle of radius $r_{B}$, as shown in Fig. 2 .

The following is a centering algorithm that will determine the values $\left(\theta_{A}, \theta_{B}\right)$ required to center on the $\mathrm{QD}$ a spot which is misaligned by $\left(\Delta x_{e}, \Delta y_{e}\right)$, where $\left(\theta_{A}, \theta_{B}\right)$ are the angular rotational displacement of RBS $A$ and $B$, respectively. A centered spot has misalignment error $(0,0)$. 


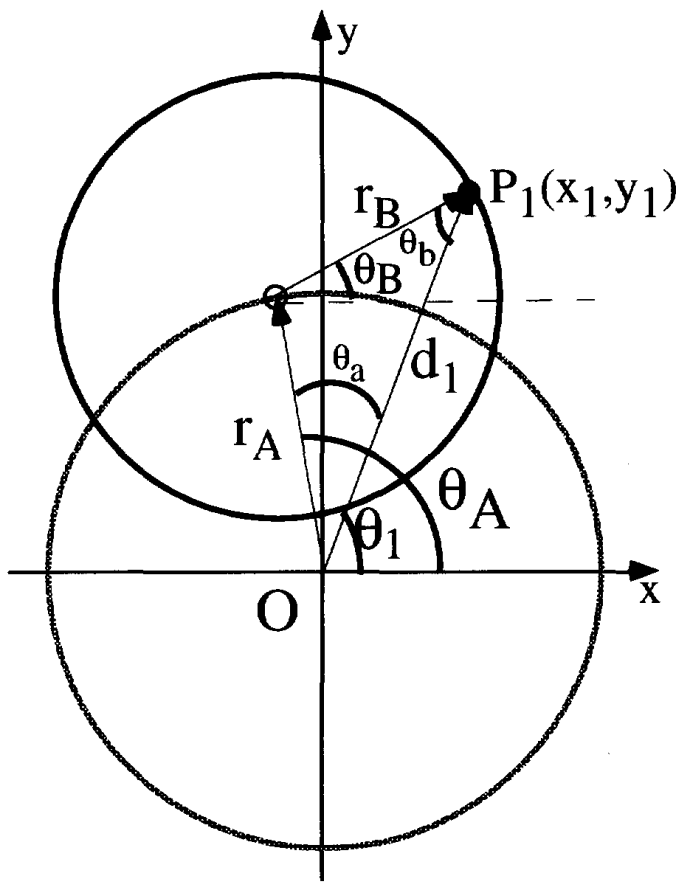

Fig. 2. Locus of spot movement on detector plane along with mathematical constructions used in derivation of transforms in centering algorithm.

1) Determine $\left(x_{u}, y_{u}\right)$, the displacement of the spot had the beam not been deviated by the RBS's, where $\left(\theta_{A}^{\prime}, \theta_{B}^{\prime}\right)$ are the previous values of $\left(\theta_{A}, \theta_{B}\right)$.

$$
\begin{aligned}
& x_{u}=\Delta x_{e}-\left(r_{A} \cos \theta_{A}^{\prime}+r_{B} \cos \theta_{B}^{\prime}\right) \\
& y_{u}=\Delta y_{e}-\left(r_{A} \sin \theta_{A}^{\prime}+r_{B} \sin \theta_{B}^{\prime}\right) .
\end{aligned}
$$

2) Determine $\left(\theta_{A}, \theta_{B}\right)$ necessary for the RBS's to impart a displacement of $\left(-x_{u},-y_{u}\right)$. The Cartesian to Risley (CtoR transform) maps $\left(-x_{u},-y_{u}\right)$ to $\left(\theta_{A}, \theta_{B}\right)$ such that $d_{1}^{2}=x_{u}^{2}+y_{u}^{2}, \theta_{1}=\arctan \left(-y_{u} /-x_{u}\right)$ where $0 \leq \theta_{1}<2 \pi$, and

$$
\begin{aligned}
& \theta_{a}=\arccos \left(\frac{r_{B}^{2}-r_{A}^{2}-d_{1}^{2}}{-2 r_{A} d_{1}}\right) \\
& \theta_{b}=\arccos \left(\frac{r_{A}^{2}-r_{B}^{2}-d_{1}^{2}}{-2 r_{B} d_{1}}\right) .
\end{aligned}
$$

The new value of $\left(\theta_{A}, \theta_{B}\right)$ is thus: $\theta_{A}=\theta_{1}+\theta_{a}$ and $\theta_{B}=\theta_{1}-\theta_{b}$

$\left(\theta_{A}, \theta_{B}\right)$ is now the desired input pair of angles to be fed to the stepper motor system controlling the RBS's. If more than one solution is possible, the algorithm chooses the solution consistent with the geometry of Fig. 2.

There are restrictions on $d_{1}:$ 1) $d_{1}<r_{A}+r_{B}$ and 2) $d_{1}>\left|r_{A}-r_{B}\right|$. The first condition implies that the total misalignment distance, $d_{1}$, must be less than the total radius of action of the RBS's. The second condition is more problematic and implies that there is a "blind area" to which the RBS's cannot center a spot. This blind area does not imply that the mismatched RBS's never will be able to center a spot,

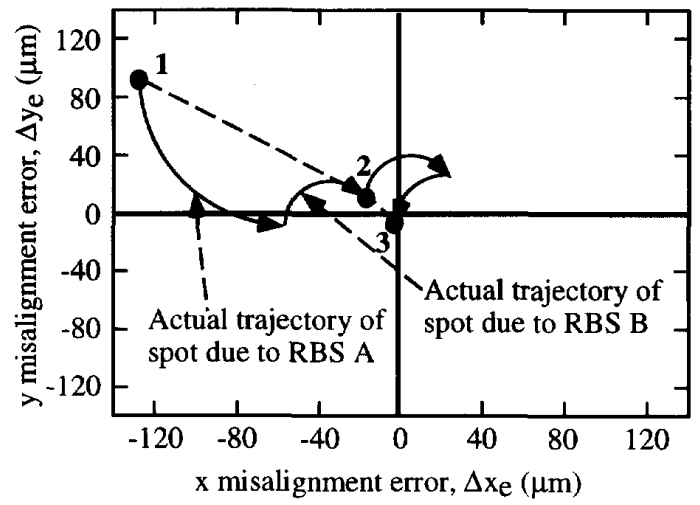

Fig. 3. Typical centering run. The solid curves indicate the actual trajectory of the spot due to the movement of the RBS's.

but rather that the RBS's never will be able to impart a net displacement of zero $\mu \mathrm{m}$ to the spot. The corollary of this statement is: only if the QD center is more than $\left|r_{A}-r_{B}\right|$ away from the optimal optical axis can the spot be centered, where the optimal optical axis is defined by a perfectly aligned system with no RBS's. In the future, matched wedges obtained by cutting one RBS in two along its diameter will be investigated.

Initial conditions must also be considered. Step 1 of the algorithm assumes the previous values of $\left(\theta_{A}, \theta_{B}\right)$ are known. However, the algorithm is only valid after an initialization routine ("hunting") determines the initial angular displacement of the RBS's. Given an unknown initial angular displacement $\left(\theta_{A}^{\prime}, \theta_{B}^{\prime}\right)$ of the RBS's and an arbitrary $\left(\Delta x_{e}^{\prime}, \Delta y_{e}^{\prime}\right)$, the hunting process rotates both RBS's until the spot's $\Delta x_{e}$ coordinate is at a maximum (maximum deflection to the right). By definition, this angular position corresponds to $\left(\theta_{A}, \theta_{B}\right)=$ $(0,0)$.

\section{EXPERIMENTAL SETUP}

The physical values used in the demonstrator were as follows: $f=100 \mathrm{~mm}, \beta_{A}=0.208^{\circ}$ and $\beta_{B}=0.278^{\circ}$, and the QD was a Hamamatsu $\$ 4349$. The minimum spot size for this system was calculated to be $3 w=15.5 \mu \mathrm{m}$ (99\% power). Since the $S 4349$ is large relative to the minimum spot size (each active area, or quadrant, on the QD is $1450 \times 1450 \mu \mathrm{m}$ with a $100 \mu \mathrm{m}$ gap between adjacent quadrants), the beam was defocused on the QD such that the spot size was $3 w \sim$ $585 \mu \mathrm{m}$ ( $99 \%$ power). This permitted a greater range for error detection, although at the expense of measurement sensitivity. The lens and a custom holder for the RBS's were installed on a Spindler and Hoyer microbench setup built around 48step stepper motors and a $60: 1$ worm gear reduction system which rotated the RBS's. With a 10 volt applied reverse bias voltage, the generated photocurrents produced voltage swings across $330 \mathrm{k} \Omega$ resistors of $0-10 \mathrm{~V}$ which were sampled by a National Instruments Lab-NB ${ }^{\mathrm{TM}}$ I/O board on a Macintosh; the algorithm was encoded on LabView ${ }^{\mathrm{TM}}$ running on the Macintosh. The voltage pulses for turning the motors were sent out by the Lab-NB ${ }^{M}$. A UCN 5804 chip between the board and motors provided power gain for the motors. 


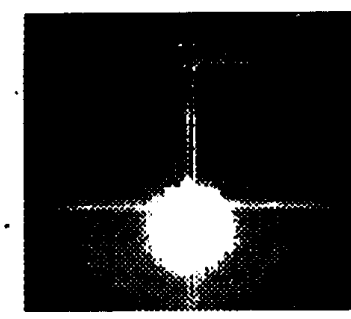

(a)

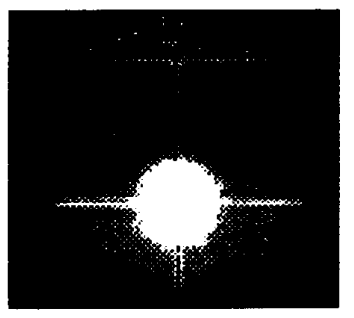

(b)
Fig. 4. (a) and (b) spot before and after a centering run. Initially, the spot was manually misaligned to position $(-213,-7) \mu \mathrm{m}$. After one iteration, the spot was at $(-8,20) \mu \mathrm{m}$. (Image is inverted and reflected by imaging system).

\section{RESULTS}

Fig. 3 shows a typical run. The spot center started at position $1,\left(\Delta x_{f} . \Delta y_{e}\right)=(-127,92) \mu \mathrm{m}$. After one iteration, the spot center was moved to position 2 . The error in centering was primarily due to the combined effect of laser fluctuations, mechanical backlash, and measurement errors occurring when the spot is far from the QD center. A second iteration brought the spot center to position 3. After this run, the QD, which was attached to an $x-y-z$ positioner, was manually moved $\sim 100 \mu \mathrm{m}$ in the $-x$ direction, to position $\left(\Delta x_{e}, \Delta y_{e}\right)=$ $(109,5) \mu \mathrm{m}$. Such an act simulated a mechanical or other perturbation. After this perturbation, the demonstrator once again centered the spot. Table I shows the result of eight consecutive centerings. In each case, the spot was aligned to within $\sim 20 \mu \mathrm{m}$ in one iteration and to within $10 \mu \mathrm{m}$ in two iterations. The measurement accuracy of the system near the origin was $\pm 5 \mu \mathrm{m}$ for each coordinate $\left(\Delta x_{e}, \Delta y_{e}\right)$, and mechanical backlash of the gears and stepper motors caused an uncertainty of $\pm 2 \mu \mathrm{m}$ in the spot position. Fig. 4 shows a spot before and after a typical steady-state centering run.

\section{FUTURE DIRECTIONS}

This active alignment demonstrator can be scaled to systems having many interconnects. For example, QD's may be fabricated on a device array and used with additional "alignment" beams to obtain positional information. Additionally, for a lenslet-array-based system [5], the lenslets imaging the alignment beams could have a different focal length from the signal
TABLE I

Result of Consecutive Centering Operations

(All Distances and Positions in Microns)

\begin{tabular}{|c|c|c|c|c|}
\hline Initial $\left(\Delta x_{e}, \Delta y_{e}\right)$ & Inivial distance to center & Final $\left(\Delta x_{e}, \Delta y_{e}\right)$ & Final distance w center & iterations \\
\hline$(-127,92)$ & 157 & $(-3.8,-6)$ & 7.1 & 2 \\
\hline$(109,5)$ & 109.1 & $(-0.8,-2)$ & 2.15 & 2 \\
\hline$(-53,-20)$ & 56.64 & $(1.1,-6.1)$ & 6.2 & 1 \\
\hline$(106,-55)$ & 119.4 & $(7.2,-1.4)$ & 7.3 & 2 \\
\hline$(-117,-89)$ & 147 & $(5,-2.9)$ & 5.8 & 2 \\
\hline$(-85,19)$ & 87.1 & $(-2,-5)$ & 5.4 & 1 \\
\hline$(90,103)$ & 136 & $(7.1,6.8)$ & 9.8 & 1 \\
\hline$(89,76)$ & 117 & $(-1.3,3.9)$ & 4.1 & 3 \\
\hline
\end{tabular}

beams', or be astigmatic in order to provide information about misalignment in the $z$-direction $\Delta z_{\mathrm{e}}$ [3]. Therefore, one QD would yield information about $\Delta x_{e}, \Delta y_{e}, \Delta z_{e}$. An additional $\mathrm{QD}$ would yield information about rotational errors.

\section{CONCLUSION}

An $x-y$ active alignment system using Risley Beam Steerers to center a spot on a quadrant detector was successfully designed, built and tested. Future research will involve scaling the system to arrays of interconnects, correcting for other types of misalignment errors, and developing better algorithms.

\section{REFERENCES}

[1] T. C. Banwell, R. C. Estes, S. F. Habiby, G. A. Hayward, T. K. Helstern, G. R. Lalk, D. D. Mahoney, D. K. Wilson, and K. C. Young, "Physical design issues for very large ATM switching systems," IEEE J. Select. Areas Commun., vol. 9, pp. 1227-1238. 1991.

[2] F. B. McCormick. T. J. Cloonan, A. L. Lentine, J. M. Sasian, R. L. Morrison, M. G. Beckman, S. L. Walker, M. J. Wojcik, S. J. Hinterlong R. J. Crisci, R. A. Novotny, and H. S. Hinton, "Five-stage free-space optical switching network with field-effect transistor self-electro-opticeffect-device smart-pixel arrays," Appl. Opt., vol. 33, pp. 1601-1618, 1994

[3] W. H. Lee, "Holographic head for compact disk applications," Opt. Eng., vol. 28 , pp. $650-653,1989$.

[4] Y. Netzer, "Line-of-Sight steering and stabilization," Opt. Eng., vol. 21, pp. $96-104,1982$

[5] B. Robertson, W. M. Robertson, G. C. Boisset, H. S. Hinton, Y. S. Liu, N. H. Kim, M. R. Otazo, D. Pavlasek, and D. V. Plant, "Design of a lenslet array based free-space optical backplane demonstrator," in Proc: Conf. Opt. Comput. paper WP19. 1994. 